Research Article

\title{
Metformin Ameliorates A $\beta$ Pathology by Insulin-Degrading Enzyme in a Transgenic Mouse Model of Alzheimer's Disease
}

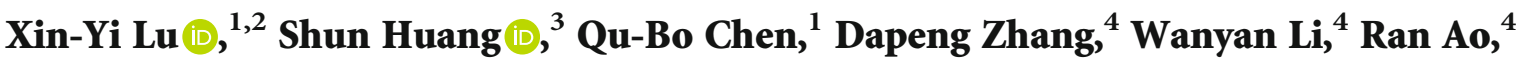 \\ Feona Chung-Yin Leung, ${ }^{5}$ Zhimin Zhang, Jisheng Huang $\mathbb{D}^{4},{ }^{6,7,8}$ Ying Tang $\mathbb{D},{ }^{9}$ \\ and Shi-Jie Zhang $\mathbb{D}^{2}$ \\ ${ }^{1}$ Biological Resource Center, The Second Affiliated Hospital of Guangzhou University of Chinese Medicine, Guangzhou, China \\ ${ }^{2}$ Department of Neurology, The Second Affiliated Hospital of Guangzhou University of Chinese Medicine, Guangzhou, China \\ ${ }^{3}$ Nanfang PET Center, Nanfang Hospital, Southern Medical University, Guangzhou, China \\ ${ }^{4}$ The First Affiliated Hospital of Guangzhou Medical University, Guangzhou, China \\ ${ }^{5}$ School of Chinese Medicine, LKS Faculty of Medicine, The University of Hong Kong, Hong Kong \\ ${ }^{6}$ Drug Non-Clinical Evaluation Center of Guangzhou Institute of Pharmaceutical Industry, Guangzhou General Pharmaceutical \\ Research Institute Co. Ltd., Guangzhou, China \\ ${ }^{7}$ School of Basic Medical Sciences, Center for Post-Doctoral Studies of Southern Medical University, Guangzhou, China \\ ${ }^{8}$ Post-Doctoral Research Center of Guangzhou Pharmaceutical Holdings Ltd., Guangzhou, China \\ ${ }^{9}$ The First Affiliated Hospital of Guangzhou University of Chinese Medicine, Guangzhou University of Chinese Medicine, \\ Guangzhou, China
}

Correspondence should be addressed to Jisheng Huang; ziyuanhuang@163.com, Ying Tang; 18825144748@163.com, and Shi-Jie Zhang; zsj19891122@gmail.com

Received 29 January 2020; Revised 5 March 2020; Accepted 25 March 2020; Published 21 April 2020

Academic Editor: Luciano Saso

Copyright (c) $2020 \mathrm{Xin}-\mathrm{Yi} \mathrm{Lu}$ et al. This is an open access article distributed under the Creative Commons Attribution License, which permits unrestricted use, distribution, and reproduction in any medium, provided the original work is properly cited.

\begin{abstract}
Alzheimer's disease $(\mathrm{AD})$ is the most common neurodegenerative disease. The accumulation of amyloid beta $(\mathrm{A} \beta)$ is the main pathology of AD. Metformin, a well-known antidiabetic drug, has been reported to have AD-protective effect. However, the mechanism is still unclear. In this study, we tried to figure out whether metformin could activate insulin-degrading enzyme (IDE) to ameliorate $\mathrm{A} \beta$-induced pathology. Morris water maze and $\mathrm{Y}$-maze results indicated that metformin could improve the learning and memory ability in $\mathrm{APP}^{\mathrm{swe}} / \mathrm{PS} 1{ }^{\mathrm{dE} 9}$ (APP/PS1) transgenic mice. ${ }^{18} \mathrm{~F}$-FDG PET-CT result showed that metformin could ameliorate the neural dysfunction in APP/PS1 transgenic mice. PCR analysis showed that metformin could effectively improve the mRNA expression level of nerve and synapse-related genes (Syp, $N g f$, and $B d n f$ ) in the brain. Metformin decreased oxidative stress (malondialdehyde and superoxide dismutase) and neuroinflammation (IL-1 $\beta$ and IL-6) in APP/PS1 mice. In addition, metformin obviously reduced the $\mathrm{A} \beta$ level in the brain of APP/PS1 mice. Metformin did not affect the enzyme activities and mRNA expression levels of $\mathrm{A} \beta$-related secretases (ADAM10, BACE1, and PS1). Meanwhile, metformin also did not affect the mRNA expression levels of $\mathrm{A} \beta$-related transporters (LRP1 and RAGE). Metformin increased the protein levels of p-AMPK and IDE in the brain of APP/PS1 mice, which might be the key mechanism of metformin on AD. In conclusion, the well-known antidiabetic drug, metformin, could be a promising drug for AD treatment.
\end{abstract}

\section{Introduction}

Alzheimer's disease $(\mathrm{AD})$, a progressive neurodegenerative disease with a high incidence rate in this century, has cognitive and functional ability decline with the disease progress. $\mathrm{AD}$ is mainly characterized by the accumulation of amyloid beta $(\mathrm{A} \beta)$ plaques and neurofibrillary tangles (NFTs) in the brain [1-3]. Accumulation of $A \beta$, which is generated from the amyloid precursor protein (APP), is the main hallmark of $\mathrm{AD}$ [4]. Thus, targeting $\mathrm{A} \beta$ is leading to a potential therapeutic strategy [5]. However, a series of clinical trials, such as inhibitors of $\beta$-secretase (BACE1) or $\gamma$-secretase (PS1), have 
failed $[6,7]$. Promoting the degradation or clearance of $A \beta$ is considered as an alternative therapeutic strategy [8].

Diabetes mellitus (DM) is also associated with a higher risk of $\mathrm{AD}[9,10]$, in which insulin deficiency or insulin resistance may be responsible. Metformin, as a major antidiabetic drug, has been demonstrated to reduce $\beta$-secretase activity, promote phospho-thr-231-tau degradation, and influence mitochondrial function [10-14]. Recently, some molecules, which are widely studied in diabetes, have been proved to affect $\mathrm{A} \beta$ clearance, including insulin-degrading enzyme (IDE), neprilysin (NEP), receptor for advanced glycation end products (RAGE), and matrix metalloproteinases (MMPs) [15-19]. Among these molecules, IDE plays an important role in both diabetes and AD. Loss-of-function mutation in the IDE gene can lead to impaired degradation of $\mathrm{A} \beta$ [20]. Colocalization and codeposition of IDE with $\mathrm{A} \beta$ plaques can be found in the $\mathrm{AD}$ brain. Inhibition of IDE in the brain is identified as one of the major factors involved in the crosstalk between DM and AD [21]. However, whether metformin could protect against AD through the IDE pathway is still unknown.

$\mathrm{APP}^{\mathrm{swe}} / \mathrm{PS}^{\mathrm{dE9}}$ (APP/PS1) double transgenic mice were used for the study. The APP/PS1 mouse exhibits the A $\beta$ plaque formation and memory impairment, which is similar to clinical phenotype [22], including memory deficits, anxiety, hyperactivity, and social interaction impairment. Metformin, an experimental therapy, was used to explore the hypothesis that metformin protects against $\mathrm{AD}$ via IDE signaling in APP/PS1 mice.

\section{Material and Methods}

2.1. Materials. Metformin and Thioflavin $\mathrm{T}(\mathrm{ThT})$ reagent were purchased from Sigma-Aldrich (Saint Louis, MO, USA). A BCA protein assay kit and superoxide dismutase (SOD) assay kit were obtained from Beyotime Biotechnology. The malondialdehyde (MDA) Assay Kit (TBA method) was purchased from Nanjing Jiancheng Bioengineering Institute (Nanjing, China). qPCR reagents and ECL kit were purchased from Invitrogen. All the antibodies were obtained from Cell Signaling Technology, Inc. (Danvers, MA, USA).

2.2. Animals and Treatments. The 7-month-old male APP/PS1 double transgenic mice and wild-type mice (C57BL/6) were obtained from the Model Animal Research Institute of Nanjing University (Nanjing, China) and maintained at the laboratory animal center of Guangzhou Medical University under the standard housing conditions with free access to food and water. The animal management was approved by the Institutional Animal Care and Use Committee of Guangzhou Medical University (SCXK20190013). All mice were randomly divided into three groups: wild type (WT, $n=15)$, APP/PS1 $(n=15)$, and APP/PS1 + metformin $(200 \mathrm{mg} / \mathrm{kg} /$ day, $n=15)[23,24]$. After oral drug administration for 8 weeks, mice were performed behavioral tests and subsequently sacrificed for the collection of brains.

2.3. Morris Water Maze Test. The Morris water maze test was performed to evaluate the spatial memory performance. The opaque platform with a diameter of $10 \mathrm{~cm}$ was positioned $1 \mathrm{~cm}$ beneath the water surface. The duration of training and testing session was $60 \mathrm{~s}$. In the training session, mice completed four trials daily with at least 20 minutes of interval between two trials for six consecutive days. Each mouse was released into the water by facing the wall in one of the four quadrants. If the mouse failed to reach the platform within $60 \mathrm{~s}$, it would be directed to the platform and stay there for $15 \mathrm{~s}$. In the testing session, the platform was removed from the pool and the mice were allowed to search for the platform for $60 \mathrm{~s}$. A computerized video imaging analysis system (Feidi, Guangzhou) was used to record and analyze the swimming paths in the maze.

2.4. Y-Maze Test. The Y-maze test was performed to evaluate the working memory performance. The apparatus consisted of three arms (one start arm and two goal arms) of $30 \times 10 \times 20 \mathrm{~cm}$ connected by an intersection. The duration of training and testing session was $2 \mathrm{~min}$. Before the training and testing sessions, the body weight of mice would reduce to $90 \%$ by food restriction. In the training session, mice completed 10 trials daily with at least 20 min interval between two trials for four consecutive days. Prior to the reward alternation testing session, each mouse would consume the food which was filled at the end of the arm for 4-6 times to habituate to the maze. The choice for each goal arm should be given equally, and the percentage of correct choices would be calculated to analysis.

2.5. ${ }^{18}$ F-FDG PET Imaging. After 8 weeks of metformin administration, the microPET-CT was used to evaluate brain glucose uptake. ${ }^{18} \mathrm{~F}$-Fluordeoxyglucose $\left({ }^{18} \mathrm{~F}\right.$-FDG) was intraperitoneally injected into animals. The mice were scanned by using a Focus 220 microPET scanner (Siemens Medical Solutions USA, Inc., Knoxville, TN, USA). Dynamic scans were conducted for $1 \mathrm{~h}$. PET images were reconstructed using the microPET-CT manager (Siemens Medical Solutions USA, Inc.). To evaluate relative glucose metabolism, the ratio of the SUV standardized uptake value was obtained by dividing the SUV of each region with the SUV of the whole brain.

2.6. ELISA. Brain tissues were homogenized with saline, containing a cocktail of protease inhibitors. Samples were centrifuged by $12000 \times g$ for $15 \mathrm{~min}$. The supernatants were measured for IL-1 $\beta$, IL-6 (Thermo Fisher Scientific), A $\beta 1$ 40, A $\beta 1-42$ (Invitrogen), and $\alpha-, \beta$-, and $\gamma$-secretases (R\&D Systems, USA) by using the ELISA kits according to the manufacturer's proposals.

2.7. ThT Staining. The sections were washed three times in PBS at room temperature and then incubated with a ThT reagent $(50 \mu \mathrm{M})$ for $30 \mathrm{~min}$. After washing three times in PBS, the sections were captured by a fluorescence microscope (Leica).

2.8. Measurement of SOD and MDA. The brain tissues were homogenized and centrifuged by $12000 \times g$ for $15 \mathrm{~min}$. The supernatants were used to test SOD activity and MDA level according to the kit instructions (Nanjing Jiancheng Bioengineering Institute). 
2.9. $q P C R$. Total RNA from brain tissues were extracted using a TRIzol reagent. Reverse transcription was treated with an ExScript RT Reagent Kit (Invitrogen). Real-time PCR analysis was undertaken using SYBR Premix Ex Taq (Invitrogen). The transcriptions were investigated for several target genes, including synaptophysin (Syp): For $5^{\prime}$-GTGCTGCAATG GGTCTTCG- $3^{\prime}$ and Rev $5^{\prime}$-CCGTGGCCAGAAAGTC CAG-3'; nerve growth factor (Ngf): For $5^{\prime}$-CAAGGACGC AGCTTTCTATACTG- ${ }^{\prime}$ and Rev $5^{\prime}$-CTTCAGGGACA GAGTCTCCTTCT-3'; brain-derived neurotrophic factor $(B d n f)$ : For $5^{\prime}$-TACTTCGGTTGCATGAAGGCG-3' ${ }^{\prime}$ and Rev 5'-GTCAGACCTCTCGAACCTGCC-3'; a disintegrin and metalloproteinase domain-containing protein 10 (ADAM10): For 5' -TTCTCCCTCCGGATCGATGT-3' and Rev $5^{\prime}$-ATACTGACCTCCCATCCCCG-3'; beta-secretase 1 (BACE1): For $5^{\prime}$-ACTTTACACTCTGTTCTGGGTGG-3' and Rev $5^{\prime}$-ACCACAAAGCCTGGCAATCTC-3'; presenilin 1 (PS1): For $5^{\prime}$-AATGACGACAACGGTGAGGG-3' and Rev $5{ }^{\prime}$-CCAGATTAGGTGCTTCCCCG-3'; low-density lipoprotein receptor-related protein 1 (LRP1): For $5^{\prime}$-GGAC CACCATCGTGGAAA- $3^{\prime}$ and Rev $5^{\prime}$-TCCCAGCCACG GTGATAG-3'; receptor for advanced glycation end products (RAGE): For 5'-GGACCCTTAGCTGGCACTTAGA-3' and Rev $5^{\prime}$-GAGTCCCGTCTCAGGGTGTCT-3'; and $\beta$ actin: For $5^{\prime}$-AGAGCTACGAGCTGCCTGAC-3' and Rev 5'-AGCACTGTGTTGGCGTACAG-3'.

2.10. Western Blotting. The fresh brain tissues were homogenated with RIPA buffer containing $1 \%$ phenylmethanesulfonyl fluoride and phosphatase inhibitors. The samples was separated by sodium dodecyl sulfate polyacrylamide gel electrophoresis (SDS-PAGE) and transferred onto PVDF membranes. The blotting membranes were blocked with 5\% BSA. The membranes were incubated at $4^{\circ} \mathrm{C}$ overnight with the following primary antibodies: anti-p-AMP-activated protein kinase (AMPK), anti-AMPK, anti-IDE, anti-NEP, and anti- $\beta$-actin. All the antibodies were purchased from CST. The membranes were incubated with a secondary antibody at room temperature. The membranes were covered with mixed liquid from the ECL Chemiluminescent Substrate Reagent Kit. The bands were scanned by a luminescent image analyzer (Invitrogen).

2.11. Statistical Analysis. All statistical analyses were conducted by SPSS 20.0 software. One-way analysis of variance (ANOVA) and Student $t$-test were used to analyze. Data were expressed as means \pm SEM. $p<0.05$ was considered significant.

\section{Results}

3.1. Metformin Ameliorates Learning and Memory Dysfunctions in APP/PS1 Mice. To prove the therapeutic effect of metformin on APP/PS1 mice, the Morris water maze and Y-maze tests were used to assess the cognitive function. Results showed that APP/PS1 mice suffered an obvious decline in cognitive function. Metformin could significantly improve escape latency, increase the crossing times, and shorten the time of finding the platform (Figures 1(a)-1(c)). Swimming speed differences among the three groups did not have any statistical significance (Figure 1(d)). In the Y-maze test, APP/PS1 mice showed a significant downward trend in spontaneous alternation, compared to the WT group. After metformin treatment, the mice exhibited better performance than the APP/PS1 group (Figure 1(e)).

3.2. Metformin Improves Brain Function in APP/PS1 Mice. Decreased glucose metabolism is the characteristic symptom of $\mathrm{AD} .{ }^{18} \mathrm{~F}$-FDG-PET was used to evaluate the cerebral metabolism. As shown in Figure 2, microPET-CT imaging suggested that ${ }^{18}$ F-FDG uptake intensity was sharply decreased in the brain of APP/PS1 mice. Metformin remarkably increased ${ }^{18}$ F-FDG uptake in the brain of APP/PS1 mice. In addition, the mRNA expression levels of neurotrophic factors (Bdnf and $N g f)$ and synaptic factor $(S y p)$ were significantly reduced in APP/PS1 mice (Figures 3(a)-3(c)). Metformin significantly improved the mRNA expression levels of neurotrophic factors and synapse-related proteins.

3.3. Metformin Reduces Oxidative Stress and Inflammation in the Brain of APP/PS1 Mice. Neural oxidative stress and inflammation are the key pathologies of $\mathrm{AD}$ [25]. In the brain of APP/PS1 mice, the level of MDA was increased, and the activity of SOD was reduced (Figures 4(a) and 4(b)). Metformin significantly relieved the oxidative stress status. In addition, the levels of inflammatory markers, IL-1 $\beta$ and IL-6, were significantly increased in the brain of APP/PS1 mice (Figures 4(c) and 4(d)). Metformin reduced the levels of IL$1 \beta$ and IL-6. These data suggested that metformin could inhibit oxidative stress and inflammation in APP/PS1 mice.

3.4. Metformin Reduces $A \beta$ Accumulation in the Brain of $A P P / P S 1$ Mice. The accumulation of $\mathrm{A} \beta$ is the main pathological feature of AD. The brain of APP/PS1 mice is overloaded with $\mathrm{A} \beta$. Thus, $\mathrm{A} \beta$ levels were further studied. ELISA results indicated that metformin effectively reduced the levels of $\mathrm{A} \beta 1-40$ and $\mathrm{A} \beta 1-42$ in the brain of APP/PS1 mice (Figures 5(a) and 5(b)). ThT staining results further confirmed that metformin ameliorated $\mathrm{A} \beta$ accumulation in the brain of APP/PS1 mice (Figure 5(c)). These were strong evidences that metformin could reduce $\mathrm{A} \beta$ accumulation in APP/PS1 mice.

3.5. Metformin Activates AMPK and Increases IDE in the Brain of APP/PS1 Mice. We next studied how metformin influences $A \beta$ metabolism. Sequential cleavage of the APP by $\beta$ - and $\gamma$-secretases can produce $\mathrm{A} \beta$ [26]. Thus, we firstly detected these secretases. ELISA results showed that metformin had no effect on $\alpha$-, $\beta$-, or $\gamma$-secretase (Figures 6(a)$6(\mathrm{c})$ ). The mRNA expression levels of ADAM10, BACE1, and PS1 were also tested. Metformin did not affect these gene expression levels except for a slight decrease of $B A C E 1$ (Figures 6(d)-6(f)). We next detected the A $\beta$ transportationrelated gene. Results showed that metformin also had no effect on the mRNA expression levels of LRP1 and RAGE (Figures $6(\mathrm{~g})$ and $6(\mathrm{~h})$ ). In addition, IDE and NEP, as the degrading enzymes of $\mathrm{A} \beta$, are other key aspects of the process 


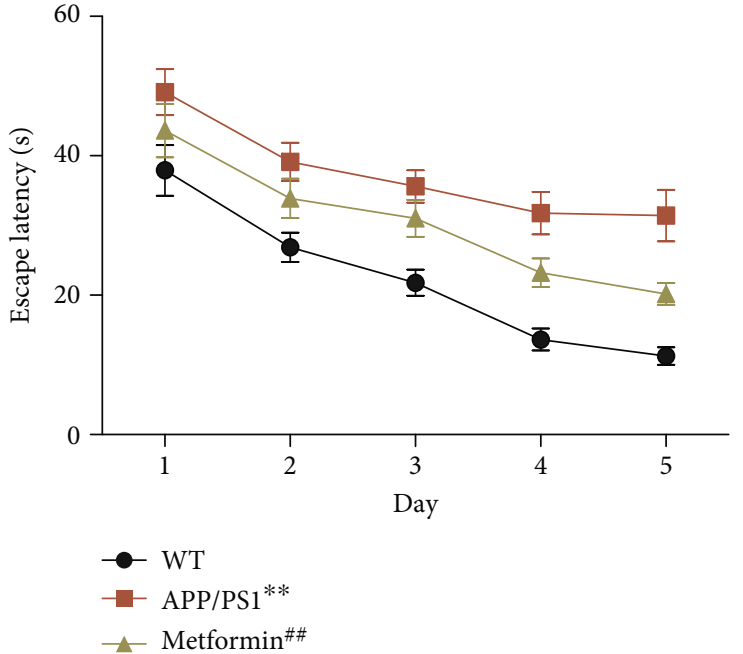

(a)

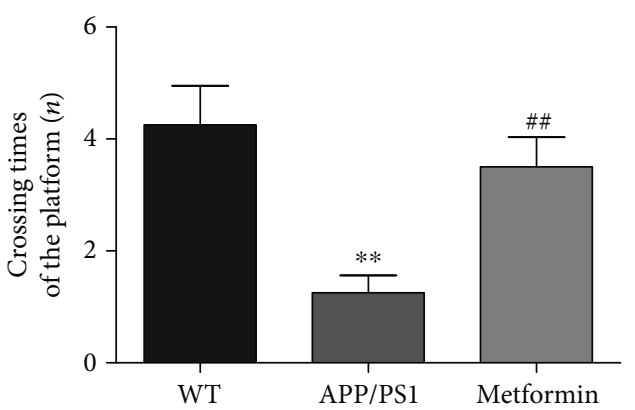

(c)

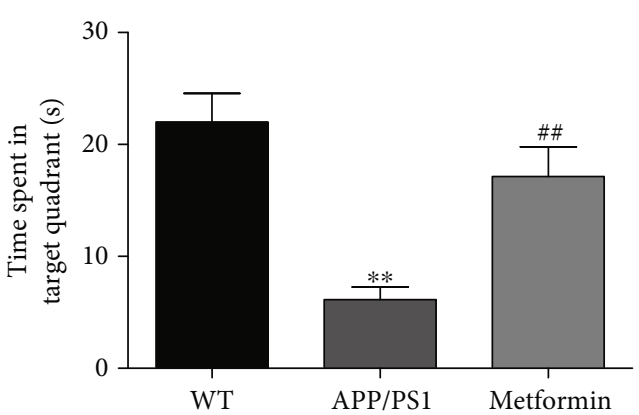

(b)

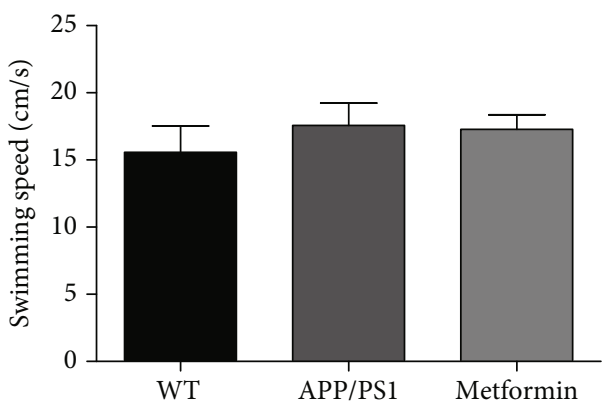

(d)

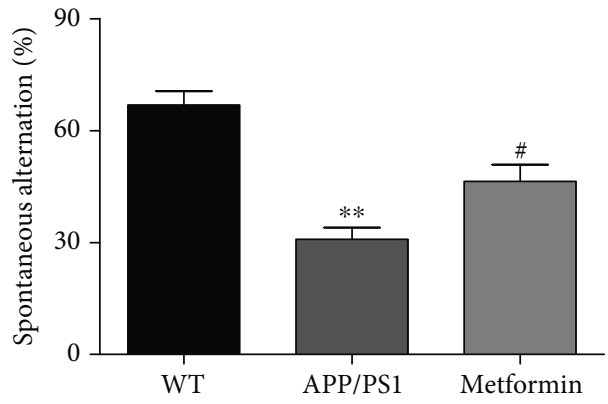

(e)

FIGURE 1: Metformin improves learning and memory impairment in APP/PS1 mice. (a) Escape latency of the five-day Morris water maze. (b) Time spent in the target quadrant in the Morris water maze. (c) Crossing times of the target platform in the Morris water maze. (d) Swimming speed in the Morris water maze. (e) Percentage of spontaneous alternation of Y-maze. Data represent the mean \pm SEM ( $n=15$ per group). ${ }^{*} p<0.05,{ }^{* *} p<0.01$, and ${ }^{* * *} p<0.001$ vs. WT; ${ }^{\#} p<0.05,{ }^{\# \#} p<0.01$, and ${ }^{\# \# \#} p<0.001$ vs. APP/PS1.

of $A \beta$ clearance. We assessed the protein expression levels of IDE and NEP in the brain of APP/PS1 mice (Figure 7). The IDE and NEP expression levels were significantly decreased in APP/PS1 mice compared to wild-type mice. Metformin significantly increased the expression level of IDE, but not NEP, in the APP/PS1 mice. Previous studies have reported that the AMPK pathway is involved in metformin effect [24, 27-30]. Our results verified this phenomenon. Metformin significantly increased the protein expression level of p-AMPK. These data suggested that the IDE signaling pathway might participate in the neuroprotective effect of metformin.

\section{Discussion}

In this study, we verified that the antidiabetic drug, metformin, could effectively ameliorate AD symptom in APP/PS1 double transgenic mice. After 8 weeks' treatment, we found that metformin could relieve learning and memory dysfunction and improve brain function. Meanwhile, metformin signally inhibited oxidative stress and neuroinflammation. Furthermore, metformin activates AMPK and increases IDE in the brain of APP/PS1 mice, which might be the key neuroprotective mechanism of metformin. 


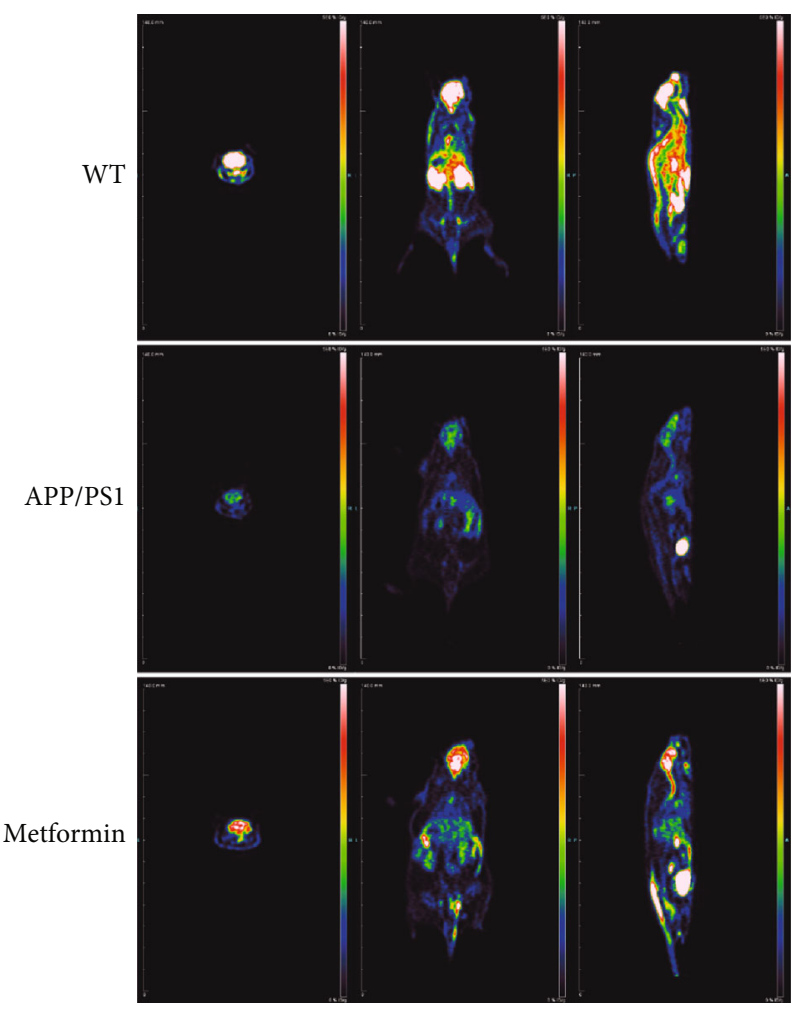

(a)

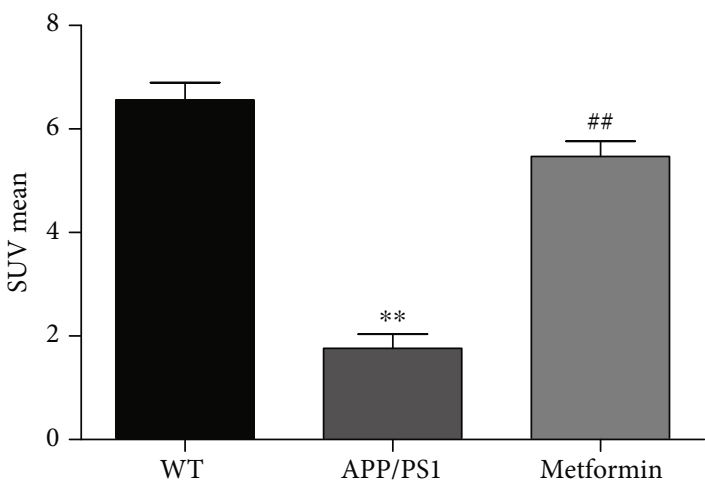

(b)

FIGURE 2: Metformin improves glucose metabolism in APP/PS1 mice. (a) PET-CT images. (b) ${ }^{18}$ F-FDG uptake of mice brains. Data represent the mean \pm SEM $\left(n=3\right.$ per group). ${ }^{*} p<0.05,{ }^{* *} p<0.01$, and ${ }^{* * *} p<0.001$ vs. WT; ${ }^{\#} p<0.05,{ }^{\# \#} p<0.01$, and ${ }^{\# \# \#} p<0.001$ vs. APP/PS1.

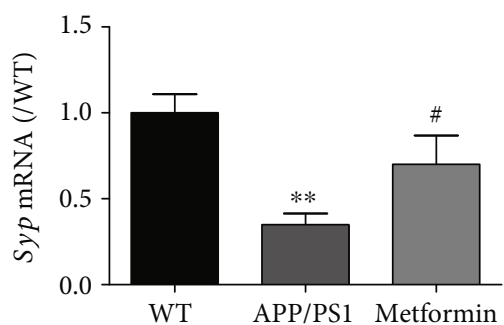

(a)

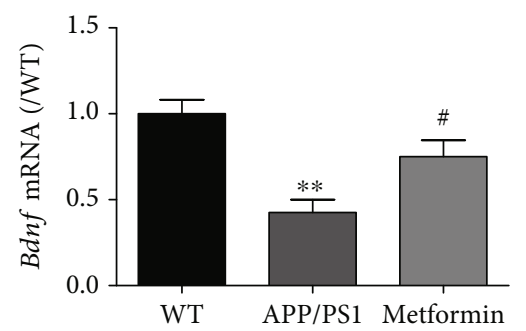

(b)

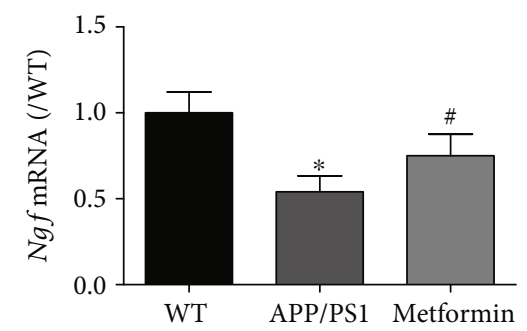

(c)

FIgURE 3: Metformin improves neurotrophic factors in APP/PS1 mice. The mRNA levels of (a) Syp, (b) Bdnf, and (c) Ngf in the APP/PS1 mice. Data represent the mean $\pm \operatorname{SEM}\left(n=6\right.$ per group). ${ }^{*} p<0.05,{ }^{* *} p<0.01$, and ${ }^{* * *} p<0.001$ vs. WT; ${ }^{\#} p<0.05$, ${ }^{\# \#} p<0.01$, and $\# \#<0.001$ vs. APP/PS1.

$\mathrm{A} \beta$ accumulation is the main pathology of $\mathrm{AD}$, which can cause the cascade reaction and induce neural apoptosis [31]. Studies have shown that metformin is beneficial for AD patients [10-12]. In this experiment, we further investigated the neuroprotective mechanism of metformin on APP/PS1 mice. We did the preliminary experiments: different dosages of metformin $(25,50,100$, and $200 \mathrm{mg} / \mathrm{kg} /$ day $)$ were given to APP/PS1 mice. Morris water maze test results indicated that metformin $(200 \mathrm{mg} / \mathrm{kg} /$ day) was the best dosage (data not shown), which was consistent with previous studies [23, 24]. Behavioral studies (Morris water maze and Y-maze) con- firmed that metformin could significantly improve learning and memory in APP/PS1 mice, which was consistent with previous studies. In addition, metformin improved cerebral metabolism and brain function and reduced the level of $A \beta$.

$\mathrm{A} \beta$ accumulation exacerbates oxidative damage and inflammation. $\mathrm{A} \beta$ can increase the synthesis of the superoxide anion, reduce the activity of catalase and SOD, activate MDA production, and finally produce reactive oxygen species [30]. In our findings, we found that the contents of MDA were significantly increased and SOD activity was significantly decreased in APP/PS1 mice. After metformin 


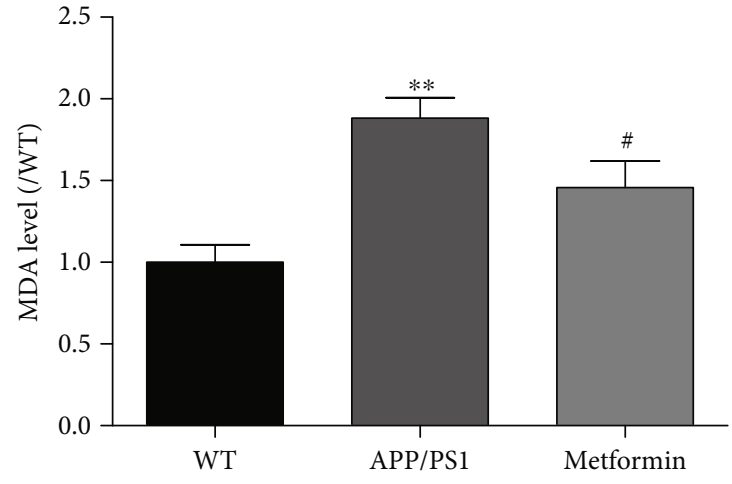

(a)

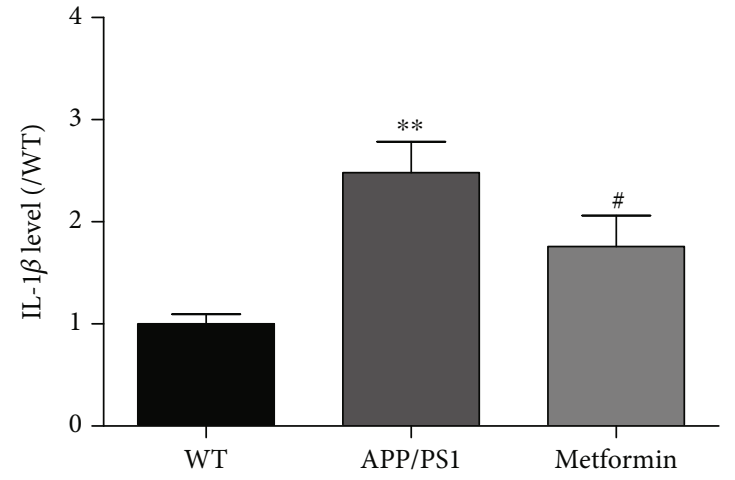

(c)

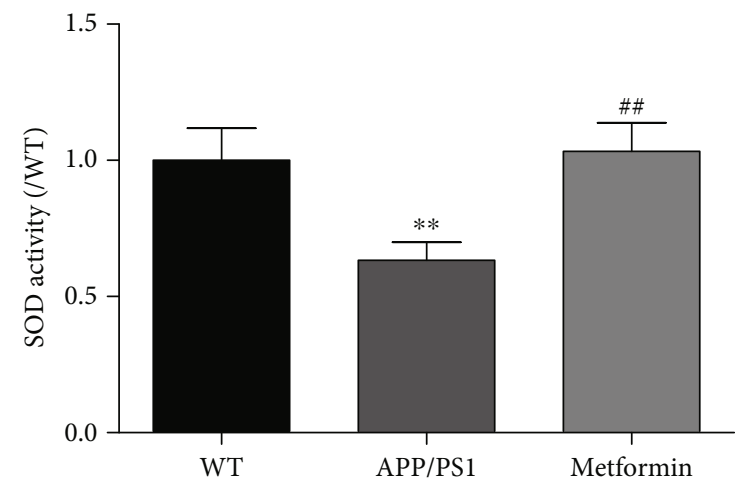

(b)

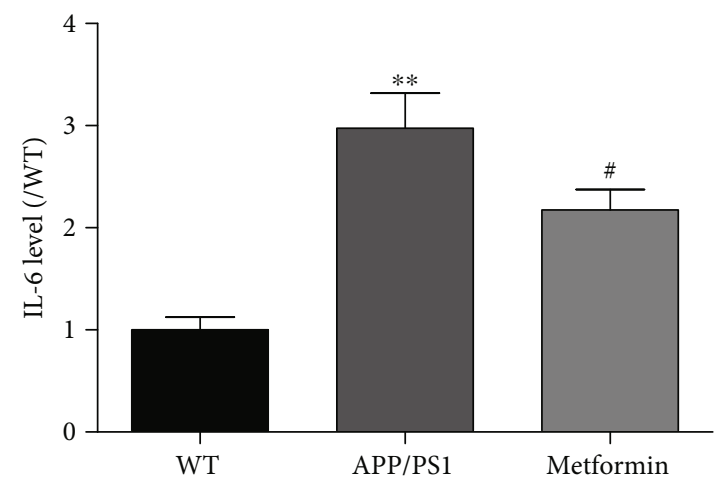

(d)

FIGURE 4: Metformin ameliorates oxidative stress and neuroinflammation in APP/PS1 mice. The level of (a) MDA and the activity of (b) SOD in the brain of APP/PS1 mice. The levels of (a) IL-1 $\beta$ and (b) IL-6 in the brain of APP/PS1 mice. Experimental values were expressed as the mean $\pm \operatorname{SEM}\left(n=6\right.$ per group). ${ }^{*} p<0.05,{ }^{* *} p<0.01$, and ${ }^{* * *} p<0.001$ vs. WT; ${ }^{*} p<0.05,{ }^{\# \#} p<0.01$, and ${ }^{\# \# \#} p<0.001$ vs. APP/PS1.

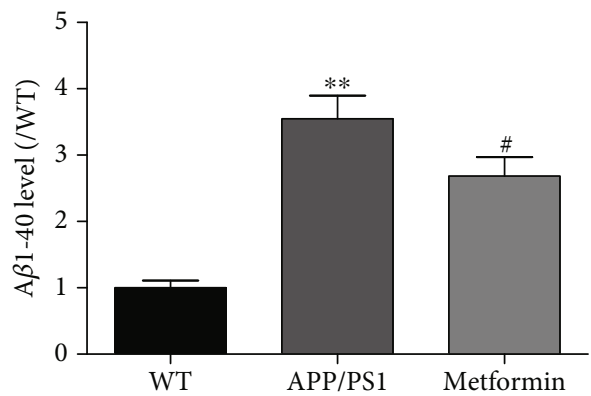

(a)

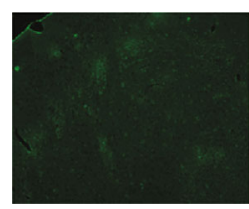

WT

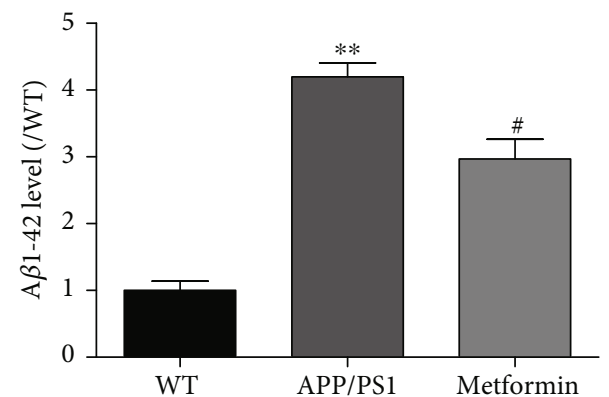

(b)

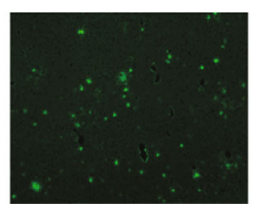

APP/PS1

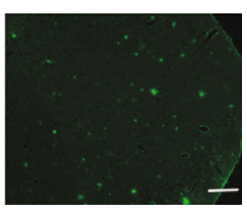

Metformin

(c)

FIgURE 5: Metformin decreases A $\beta$ levels in APP/PS1 mice. The levels of (a) A $\beta 1-40$ and (b) A $\beta 1-42$ in the brain of APP/PS1 mice. ThT staining of the brain slides in APP/PS1 mice. Experimental values were expressed as the mean \pm SEM $\left(n=6\right.$ per group). ${ }^{*} p<0.05,{ }^{* *} p<$ 0.01 , and ${ }^{* * *} p<0.001$ vs. WT; ${ }^{\#} p<0.05,{ }^{\# \#} p<0.01$, and ${ }^{\# \# \#} p<0.001$ vs. APP/PS1. Bar: $100 \mu \mathrm{m}$. 


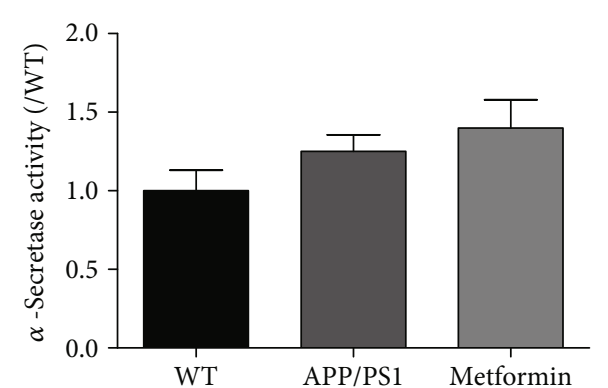

(a)

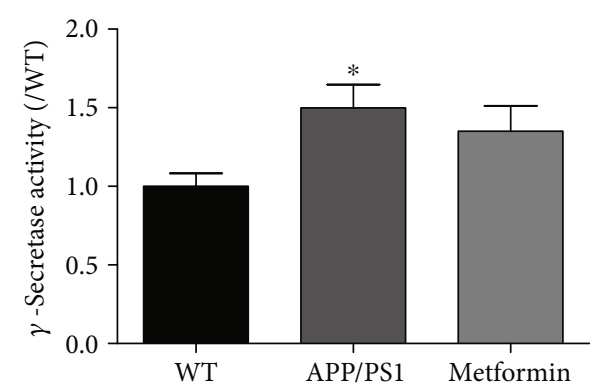

(c)

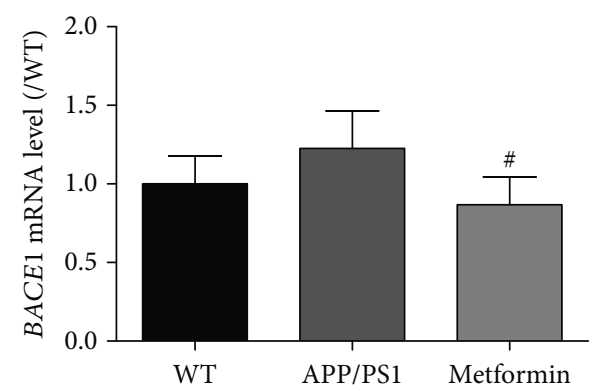

(e)

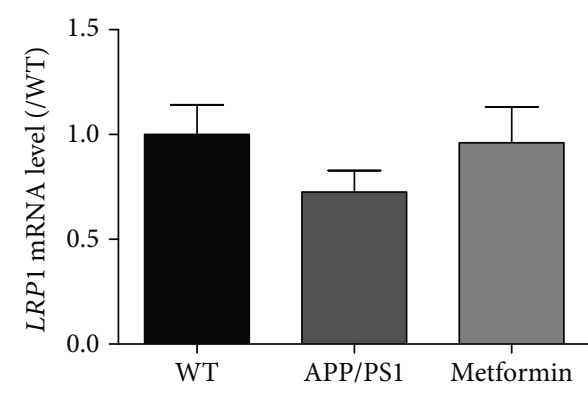

(g)

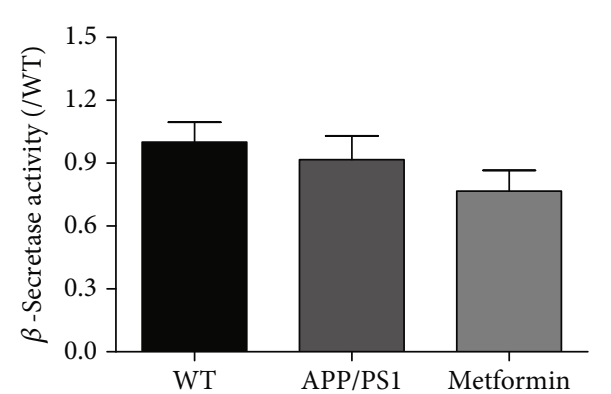

(b)

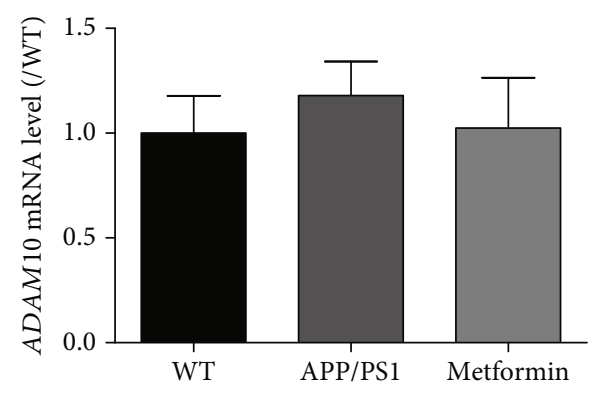

(d)

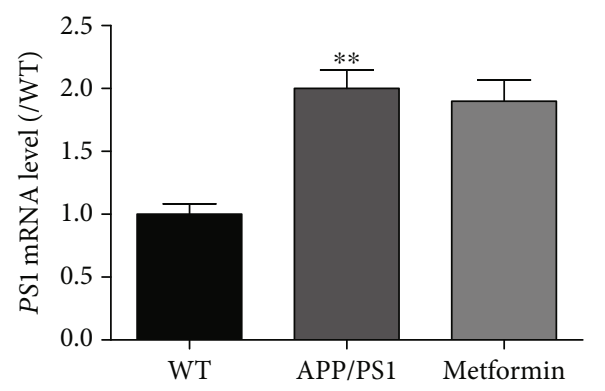

(f)

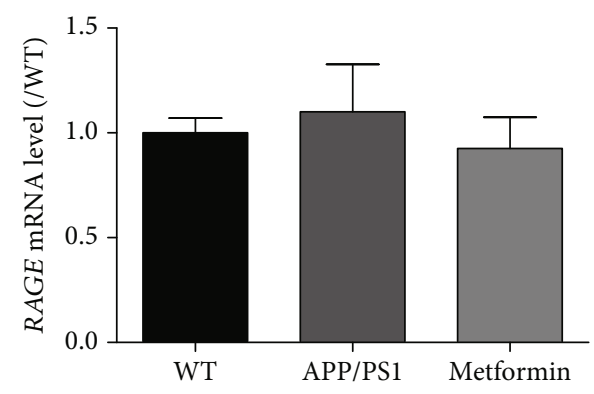

(h)

FIgURE 6: Metformin has no effect on A $\beta$ production and transportation-related genes in APP/PS1 mice. The activities of (a) $\alpha$-, (b) $\beta$-, and (c) $\gamma$-secretases. The mRNA expressions of (d) ADAM10, (e) BACE1, (f) PS1, (g) $L R P 1$, and (h) RAGE. Experimental values were expressed as the mean \pm SEM ( $n=6$ per group). ${ }^{*} p<0.05,{ }^{* *} p<0.01$, and ${ }^{* * *} p<0.001$ vs. WT; ${ }^{*} p<0.05,{ }^{\# \#} p<0.01$, and ${ }^{\# \#} p<0.001$ vs. APP/PS1.

treatment, oxidative stress was relieved. A $\beta$ accumulation can increase the levels of IL- $1 \beta$ and IL- 6 , the proinflammatory factors, in APP/PS1 mice $[32,33]$. Neuroinflammation can also exacerbate AD pathology [25]. In this study, metformin reduced the levels of IL- $1 \beta$ and IL- 6 in APP/PS1 mice.

$\mathrm{A} \beta$ production is strongly linked with $\alpha$-, $\beta$-, and $\gamma$-secretases [26]. When APP is proteolytically processed by $\beta$ - and $\gamma$-secretases, $\mathrm{A} \beta$ production is increased. When the activity of $\alpha$-secretase is increased, $\mathrm{A} \beta$ production is inhibited. $\mathrm{A} \beta$ transportation is another way of $\mathrm{A} \beta$ metabolism. RAGE and LRP1 are the two main molecules, which participate in $\mathrm{A} \beta$ transportation [34]. In this study, both ELISA and $\mathrm{qPCR}$ results showed that metformin had little influence on $\alpha$-, $\beta$-, and $\gamma$-secretases and RAGE and LRP1, except for a small reduction in BACE1 expression, which was consistent with a previous study [11]. These results indicated that except $\mathrm{A} \beta$ production and transportation, other signaling pathways might also be involved in the effect of metformin. 


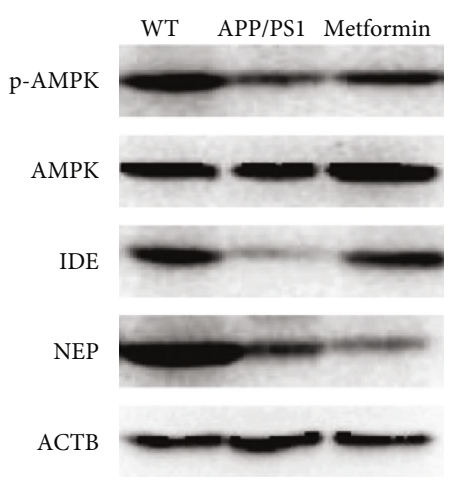

(a)

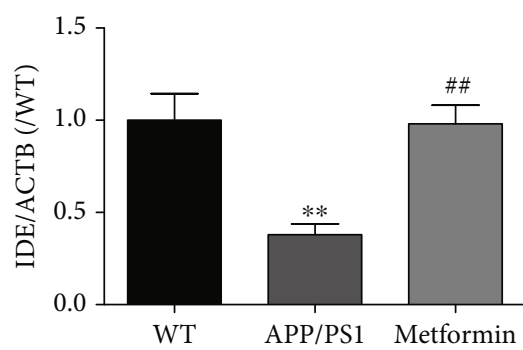

(c)

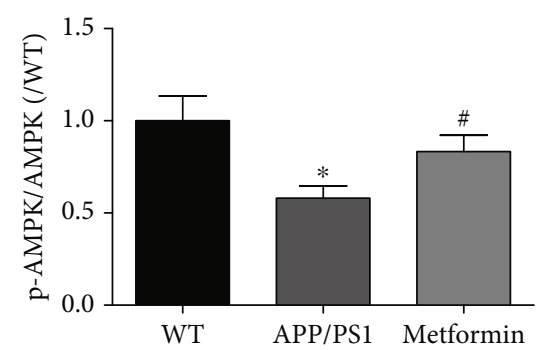

(b)

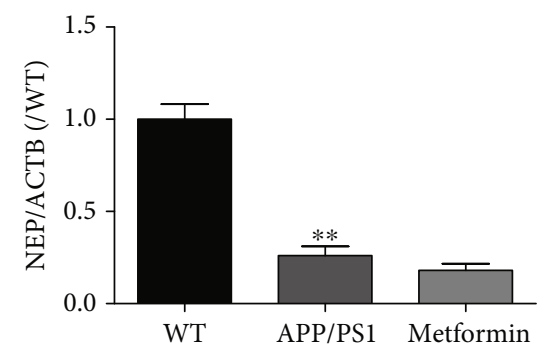

(d)

FIGURE 7: Metformin activates AMPK and increases IDE in the brain of APP/PS1 mice. (a) The representative bands of p-AMPK, AMPK, IDE, NEP, and ACTB. Western blot analysis: (b) p-AMPK/AMPK, (c) IDE/ACTB, and (d) NEP/ACTB. Experimental values were expressed as the mean \pm SEM $\left(n=3\right.$ per group). ${ }^{*} p<0.05,{ }^{* *} p<0.01$, and ${ }^{* * *} p<0.001$ vs. WT; ${ }^{\#} p<0.05,{ }^{\# \#} p<0.01$, and ${ }^{\# \# \#} p<0.001$ vs. APP/PS1.

AMPK is a crucial factor in the regulation of intracellular systems [30]. AMPK activation can enhance antiinflammatory effect, which might be regulated by AMPK $/ \mathrm{m}$ TOR and AMPK/NF- $\kappa B$ signaling pathways [24, 27-30]. Metformin was supposed to have a potential pharmacological effect, due to AMPK activation [35]. In some studies, AMPK/SIRT1 takes part in the nonamyloidogenic pathway to improve $\mathrm{AD}$ [36]. In this study, metformin obviously activated AMPK in the brain of APP/PS1 mice. In regard to A $\beta$ clearance, IDE and NEP are the main cellular degrading enzymes of $A \beta$. Early findings showed that IDE could regulate $A \beta$ and insulin levels in vivo [37]. The major locations of IDE are the cytosol, mitochondria, and peroxisomes [38]. IDE is specific toward $\beta$-structure-forming substrates of toxic oligomers $(A \beta)$ [39]. The activity of IDE in the brain decreases with age and during early stages of $\mathrm{AD}$. Overexpression of IDE in transgenic mice can prevent amyloid plaque formation [40]. Inhibition of IDE is identified as one of the crosstalk between $\mathrm{T} 2 \mathrm{D}$ and $\mathrm{AD}$ [21]. In our findings, the protein expression levels of IDE and NEP were significantly decreased in APP/PS1 mice. Metformin effectively increased the protein level of IDE. These data indicated that the IDE pathway might also participate in the neuroprotective effect of metformin.

In conclusion, we provided the evidence that metformin had beneficial effects by reducing the $\mathrm{A} \beta$ level through the IDE pathway in APP/PS1 mice. In addition, metformin could decrease inflammation and oxidative stress. However, further studies are still needed. Metformin might offer a new promising avenue in $\mathrm{AD}$ treatment.

\section{Data Availability}

The data used to support the findings of this study are available from the corresponding author upon request.

\section{Conflicts of Interest}

The authors declare that they have no conflicts of interest.

\section{Authors' Contributions}

Xin-Yi Lu, Shun Huang, and Qu-Bo Chen finished most of the experiments; Dapeng Zhang, Wanyan Li, Ran Ao, Feona Chung-Yin Leung, and Zhimin Zhang helped with data organization. Ying Tang and Shi-Jie Zhang helped to revise the manuscript. Jisheng Huang, Ying Tang, and Shi-Jie Zhang designed the experiments and modified the manuscript. Xin-Yi Lu, Shun Huang, and Qu-Bo Chen contributed equally to this work.

\section{Acknowledgments}

This work was supported by the Project of Administration of Traditional Chinese Medicine of Guangdong Province of China (Project No. 20190408212815).

\section{References}

[1] D. J. Selkoe and M. B. Podlisny, "Deciphering the genetic basis of Alzheimer's disease," Annual Review of Genomics and Human Genetics, vol. 3, pp. 67-99, 2002. 
[2] D. M. Walsh and D. J. Selkoe, "Deciphering the molecular basis of memory failure in Alzheimer's disease," Neuron, vol. 44, no. 1, pp. 181-193, 2004.

[3] Y. Yang and W. Song, "Molecular links between Alzheimer's disease and diabetes mellitus," Neuroscience, vol. 250, pp. 140-150, 2013.

[4] S. Sinha, J. P. Anderson, R. Barbour et al., "Purification and cloning of amyloid precursor protein $\beta$-secretase from human brain," Nature, vol. 402, no. 6761, pp. 537-540, 1999.

[5] P. Picone, D. Nuzzo, D. Giacomazza, and M. Di Carlo, “ $\beta$ Amyloid peptide: the cell compartment multi-faceted interaction in Alzheimer's disease," Neurotoxicity Research, vol. 37, no. 2, pp. 250-263, 2020.

[6] J. Zhang, Y. Guo, Y. Wang, L. Song, R. Zhang, and Y. Du, "Long-term treadmill exercise attenuates $\mathrm{A} \beta$ burdens and astrocyte activation in APP/PS1 mouse model of Alzheimer's disease," Neuroscience Letters, vol. 666, pp. 70-77, 2018.

[7] K. Yin, J. Jin, X. Zhu et al., "CART modulates beta-amyloid metabolism-associated enzymes and attenuates memory deficits in APP/PS1 mice," Neurological Research, vol. 39, no. 10, pp. 885-894, 2017.

[8] N. L. Sikanyika, H. C. Parkington, A. I. Smith, and S. Kuruppu, "Powering amyloid beta degrading enzymes: a possible therapy for Alzheimer's disease," Neurochemical Research, vol. 44, no. 6, pp. 1289-1296, 2019.

[9] I. Kazkayasi, M.-A.-M. Ismail, C. Parrado-Fernandez et al., "Lack of insulin results in reduced seladin-1 expression in primary cultured neurons and in cerebral cortex of STZ-induced diabetic rats," Neuroscience Letters, vol. 633, pp. 174-181, 2016.

[10] P. Picone, S. Vilasi, F. Librizzi et al., "Biological and biophysics aspects of metformin-induced effects: cortex mitochondrial dysfunction and promotion of toxic amyloid pre-fibrillar aggregates," Aging, vol. 8, no. 8, pp. 1718-1734, 2016.

[11] Y. Chen, K. Zhou, R. Wang et al., "Antidiabetic drug metformin (Glucophage ${ }^{\mathrm{R})}$ increases biogenesis of Alzheimer's amyloid peptides via up-regulating BACE1 transcription," Proceedings of the National Academy of Sciences of the United States of America, vol. 106, no. 10, pp. 3907-3912, 2009.

[12] M. P. Sajan, B. C. Hansen, M. G. Higgs et al., "Atypical PKC, $\mathrm{PKC} \lambda / l$, activates $\beta$-secretase and increases $\mathrm{A} \beta_{1-40 / 42}$ and phospho-tau in mouse brain and isolated neuronal cells, and may link hyperinsulinemia and other aPKC activators to development of pathological and memory abnormalities in Alzheimer's disease," Neurobiology of Aging, vol. 61, pp. 225237, 2018.

[13] P. Imfeld, M. Bodmer, S. S. Jick, and C. R. Meier, "Metformin, other antidiabetic drugs, and risk of Alzheimer's disease: a population-based case-control study," Journal of the American Geriatrics Society, vol. 60, no. 5, pp. 916-921, 2012.

[14] P. Picone, D. Nuzzo, L. Caruana et al., "Metformin increases APP expression and processing via oxidative stress, mitochondrial dysfunction and NF- $\kappa \mathrm{B}$ activation: use of insulin to attenuate metformin's effect," Biochimica et Biophysica Acta (BBA) - Molecular Cell ResearchBiochimica et Biophysica Acta, vol. 1853, no. 5, pp. 1046-1059, 2015.

[15] J.-M. Paumier, N. A. Py, L. García-González et al., "Proamyloidogenic effects of membrane type 1 matrix metalloproteinase involve MMP-2 and BACE-1 activities, and the modulation of APP trafficking," The FASEB Journal, vol. 33, no. 2, pp. 2910-2927, 2019.
[16] K. J. Yin, J. R. Cirrito, P. Yan et al., "Matrix metalloproteinases expressed by astrocytes mediate extracellular amyloid- $\beta$ peptide catabolism," The Journal of Neuroscience, vol. 26, no. 43, pp. 10939-10948, 2006.

[17] P. Yan, X. Hu, H. Song et al., "Matrix metalloproteinase-9 degrades amyloid- $\beta$ fibrils in vitro and compact plaques in situ," Journal of Biological Chemistry, vol. 281, no. 34, pp. 24566-24574, 2006.

[18] A. Fragkouli, E. C. Tsilibary, and A. K. Tzinia, "Neuroprotective role of MMP-9 overexpression in the brain of Alzheimer's 5xFAD mice," Neurobiology of Disease, vol. 70, pp. 179-189, 2014.

[19] K. Baranger, Y. Marchalant, A. E. Bonnet et al., "MT5-MMP is a new pro-amyloidogenic proteinase that promotes amyloid pathology and cognitive decline in a transgenic mouse model of Alzheimer's disease," Cellular and Molecular Life Sciences, vol. 73, no. 1, pp. 217-236, 2016.

[20] W. Farris, S. Mansourian, M. A. Leissring et al., "Partial lossof-function mutations in insulin-degrading enzyme that induce diabetes also impair degradation of amyloid $\beta$-protein," The American Journal of Pathology, vol. 164, no. 4, pp. 1425-1434, 2004.

[21] M. W. Akhtar, S. Sanz-Blasco, N. Dolatabadi et al., "Elevated glucose and oligomeric $\beta$-amyloid disrupt synapses via a common pathway of aberrant protein $S$-nitrosylation," Nature Communications, vol. 7, no. 1, article 10242, 2016.

[22] H. Huang, S. Nie, M. Cao et al., "Characterization of AD-like phenotype in aged APPSwe/PS1dE9 mice," Age, vol. 38, no. 4, pp. 303-322, 2016.

[23] S. A. Farr, E. Roesler, M. L. Niehoff, D. A. Roby, A. McKee, and J. E. Morley, "Metformin improves learning and memory in the SAMP8 mouse model of Alzheimer's disease," Journal of Alzheimer's Disease, vol. 68, no. 4, pp. 1699-1710, 2019.

[24] Z. Ou, X. Kong, X. Sun et al., "Metformin treatment prevents amyloid plaque deposition and memory impairment in APP/PS1 mice," Brain, Behavior, and Immunity, vol. 69, pp. 351-363, 2018.

[25] S. Kyrkanides, R. H. Tallents, J.-n. H. Miller et al., "Osteoarthritis accelerates and exacerbates Alzheimer's disease pathology in mice," Journal of Neuroinflammation, vol. 8, no. 1, article 112, 2011.

[26] F. Song, T. Liu, S. Meng, F. Li, Y. Zhang, and L. Jiang, "Insulinlike growth factor- 1 alleviates expression of $\mathrm{A} \beta_{1-40}$ and $\alpha-, \beta$, and $\gamma$-secretases in the cortex and hippocampus of APP/PS1 double transgenic mice," Journal of Molecular Neuroscience, vol. 66, no. 4, pp. 595-603, 2018.

[27] Z. Gong, J. Huang, B. Xu et al., "Urolithin A attenuates memory impairment and neuroinflammation in APP/PS1 mice," Journal of Neuroinflammation, vol. 16, no. 1, p. 62, 2019.

[28] R. Corpas, C. Grinan-Ferre, E. Rodriguez-Farre, M. Pallas, and C. Sanfeliu, "Resveratrol induces brain resilience against Alzheimer neurodegeneration through proteostasis enhancement," Molecular Neurobiology, vol. 56, no. 2, pp. 15021516, 2019.

[29] H. Zhang, C. Zhao, G. Cao et al., "Berberine modulates amyloid- $\beta$ peptide generation by activating AMP-activated protein kinase," Neuropharmacology, vol. 125, pp. 408-417, 2017.

[30] M. Markowicz-Piasecka, J. Sikora, A. Szydlowska, A. Skupien, E. Mikiciuk-Olasik, and K. M. Huttunen, "Metformin - a future therapy for neurodegenerative diseases : theme: drug discovery, development and delivery in Alzheimer's disease 
guest editor: Davide Brambilla," Pharmaceutical Research, vol. 34, no. 12, pp. 2614-2627, 2017.

[31] Y. M. Li, M. Xu, M. T. Lai et al., "Photoactivated gammasecretase inhibitors directed to the active site covalently label presenilin 1," Nature, vol. 405, no. 6787, pp. 689-694, 2000.

[32] L. Hoeijmakers, S. R. Ruigrok, A. Amelianchik et al., "Early-life stress lastingly alters the neuroinflammatory response to amyloid pathology in an Alzheimer's disease mouse model," Brain, Behavior, and Immunity, vol. 63, pp. 160-175, 2017.

[33] M. L. de Lemos, A. V. de la Torre, D. Petrov et al., "Evaluation of hypoxia inducible factor expression in inflammatory and neurodegenerative brain models," The International Journal of Biochemistry \& Cell Biology, vol. 45, no. 7, pp. 1377-1388, 2013.

[34] Q. Liu, J. Zhang, H. Tran et al., "LRP1 shedding in human brain: roles of ADAM10 and ADAM17," Molecular Neurodegeneration, vol. 4, no. 1, p. 17, 2009.

[35] R. D. Yudhani, I. Astuti, M. Mustofa, D. Indarto, and M. Muthmainah, "Metformin modulates cyclin D1 and P53 expression to inhibit cell proliferation and to induce apoptosis in cervical cancer cell lines," Asian Pacific Journal of Cancer Prevention, vol. 20, no. 6, pp. 1667-1673, 2019.

[36] S. A. Shah, G. H. Yoon, S. S. Chung et al., "Novel osmotin inhibits SREBP2 via the AdipoR1/AMPK/SIRT1 pathway to improve Alzheimer's disease neuropathological deficits," Molecular Psychiatry, vol. 22, no. 3, pp. 407-416, 2017.

[37] W. Farris, S. Mansourian, Y. Chang et al., "Insulin-degrading enzyme regulates the levels of insulin, amyloid $\beta$-protein, and the $\beta$-amyloid precursor protein intracellular domain in vivo," Proceedings of the National Academy of Sciences of the United States of America, vol. 100, no. 7, pp. 4162-4167, 2003.

[38] G. R. Tundo, D. Sbardella, C. Ciaccio et al., "Multiple functions of insulin-degrading enzyme: a metabolic crosslight?," Critical Reviews in Biochemistry and Molecular Biology, vol. 52, no. 5, pp. 554-582, 2017.

[39] I. V. Kurochkin, E. Guarnera, and I. N. Berezovsky, "InsulinDegrading Enzyme in the Fight against Alzheimer's Disease," Trends in Pharmacological Sciences, vol. 39, no. 1, pp. 49-58, 2018.

[40] A. Stargardt, J. Gillis, W. Kamphuis et al., "Reduced amyloid- $\beta$ degradation in early Alzheimer's disease but not in the APPswePS1dE9 and 3xTg-AD mouse models," Aging Cell, vol. 12, no. 3, pp. 499-507, 2013. 